Annuaire suisse de politique de développement

21 | 2002

Agriculture suisse et mondialisation

\title{
4. Développement humain
}

\section{Christoph Stamm}

\section{OpenEdition}

Journals

Édition électronique

URL : http://journals.openedition.org/aspd/943

DOI : 10.4000/aspd.943

ISSN : 1663-9669

\section{Éditeur}

Institut de hautes études internationales et du développement

\section{Édition imprimée}

Date de publication : 1 avril 2002

Pagination : 193-206

ISSN : 1660-5934

\section{Référence électronique}

Christoph Stamm, «4. Développement humain », Annuaire suisse de politique de développement [En ligne], 21 | 2002, mis en ligne le 06 septembre 2012, consulté le 07 septembre 2020. URL : http:// journals.openedition.org/aspd/943; DOI : https://doi.org/10.4000/aspd.943 


\section{DÉVELOPPEMENT HUMAIN*}

\subsection{CONFÉRENCE MONDIALE CONTRE LE RACISME À DURBAN}

Organisée par les Nations Unies et par l'Afrique du Sud (pays hôte), la $3^{e}$ "Conférence mondiale contre le racisme, la discrimination raciale, la xénophobie et l'intolérance qui y est associée" s'est tenue à Durban du 31 août au 8 septembre 2001. Réunir la conférence en Afrique du Sud, naguère encore le pays de l'apartheid, revêtait une grande importance symbolique puisque les débats sur la politique d'apartheid avaient marqué les deux conférences contre le racisme organisées jusqu'alors (en 1978 et en 1983). Des représentants de 163 pays, 12 chefs d'Etat et près de 4000 représentants d'organisations non gouvernementales ont pris part à la conférence.

L'Assemblée générale des Nations Unies désirait que la conférence s'en tienne aux faits et qu'elle s'attache à trouver des mesures concrètes pour lutter contre le racisme. La secrétaire générale de la conférence, Mary Robinson, souhaitait avant tout que les victimes prennent la parole - au nom du même droit à la parole pour tous - et que les pays ou les gouvernements à l'origine des inégalités que l'on déplore soient appelés à s'expliquer à ce sujet.

La conférence visait à adopter une déclaration finale et un programme d'action sur le racisme. Or, la longueur de son titre officiel annonçait une conférence qui devrait s'attaquer à toute une série de discriminations à condamner et à éliminer. Conformément à la volonté du comité préparatoire et de certains Etats, la conférence devait également se pencher sur les phénomènes suivants: l'attitude de rejet à l'égard des migrants, l'islamophobie, le sentiment anti-arabe, l'esclavagisme, le colonialisme et le sionisme.

$\square$ Le problème de l'indemnisation des victimes de la traite des Noirs, de l'esclavagisme et du colonialisme

L'examen de ces événements du passé a soulevé les controverses les plus vives au cours de la conférence, toutefois sans qu'elles se cristallisent en deux blocs d'avis opposés. Une majorité d'Etats africains ont demandé que le trafic d'esclaves soit classé parmi les crimes contre l'humanité et ont exigé que les pays qui ont profité pendant des siècles de la traite des Noirs vers les Amériques présentent des excuses. D'autres Etats ainsi que divers organismes de Noirs africains ou américains ont cependant refusé de se satisfaire d'un geste symbolique et ont demandé que les victimes de l'esclavage soient indemnisées. Les revendications variaient selon la provenance des demandes en réparation, celles-ci allant de l'annulation de la dette des pays africains au versement de dédommagements individuels en passant par la création d'un fonds de formation. Certains sont allés jusqu'à demander le versement d'indemnisations pour les dommages et les préjudices causés par le colonialisme. Les Etats européens impliqués dans le trafic d'esclaves ont catégoriquement refusé tout débat portant sur le versement d'indemnisations,

* Par Christoph Stamm, étudiant en sciences politiques à l'Université de Genève. 
en rappelant que la conférence avait pour but de lutter contre les formes actuelles de la discrimination. Leurs opposants ont rejeté cet argument en arguant que les conséquences néfastes de l'esclavage se faisaient sentir aujourd'hui encore.

\section{$\square$ Le conflit israélo-palestinien et l'assimilation du sionisme au racisme}

Outre le problème de l'esclavage, le sionisme a également suscité de vives controverses. Les Etats arabes et quelques Etats islamiques ont déclaré qu'Israël applique un régime d'apartheid et ont exigé que le document final de la conférence assimile expressément le sionisme au racisme. Quelques organisations non gouvernementales ont accusé Israël de crimes de guerre et lui ont reproché de pratiquer l'épuration ethnique contre le peuple palestinien. La délégation israélienne a, quant à elle, déclaré que le conflit israélo-palestinien est politique et non pas ethnique, et elle a aussi rappelé que les Juifs ont été pendant des siècles les principales victimes du racisme. Aucun compromis ne semblant envisageable, la délégation des Etats-Unis - qui avait pris fait et cause pour Israël - a quitté la conférence. Puis le ministre israélien des Affaires étrangères, Shimon Peres, a également rappelé la délégation israélienne. D'autres Etats, notamment ceux de l'Union européenne et la Norvège, se sont également opposés à la volonté d'assimiler le sionisme au racisme et ont malgré tout plaidé en faveur d'un compromis.

\section{$\square$ Le rôle des organisations non gouvernementales}

Grâce à quelques ONG, tout le bruit qui a entouré les discussions sur les deux principaux sujets controversés n'a pas complètement occulté les débats sur des formes de discrimination raciale moins criantes mais non moins graves. Des ONG indiennes ont par exemple évoqué le cas des intouchables (ou dalits): malgré l'égalité inscrite dans la Constitution indienne, pour les 170 millions de dalits qui vivent en Inde la discrimination et la violence font partie de leur lot quotidien. Ces organisations ont appelé la conférence à laisser les victimes du système des castes s'exprimer et à condamner, dans sa déclaration finale, l'«intouchabilité» comme étant un crime contre l'humanité.

Les ONG ont surtout aussi rappelé la situation indicible des femmes en Afghanistan, l'oppression des minorités chinoises, la situation des Blancs au Zimbabwe, l'esclavage au Soudan et les difficultés que rencontrent les populations indigènes d'Amérique. On a également abordé des problèmes plus généraux tels que l'application discriminatoire de la peine de mort ou les risques particuliers que courent les réfugiés et les requérants d'asile. Dans leur déclaration finale, les $\mathrm{ONG}$ ont invité les Etats à passer enfin aux actes pour mettre fin à la crise des droits de l'homme.

Lors des votes, quelques ONG se sont cependant montrées très intolérantes envers certains Etats ou communautés; elles n'ont ainsi fait qu'accroître les tensions et rendu difficile la recherche de compromis.

\section{$\square$ La position de la Suisse}

La délégation suisse, qui comptait huit membres, était emmenée par Claudia Kaufmann, secrétaire générale du Département fédéral de l'intérieur et cheffe de la délégation à la Conférence préparatoire. 
La Suisse a observé une grande réserve quant aux thèmes vivement controversés et, mettant l'accent sur ses préoccupations «techniques», elle a surtout œuvré pour promouvoir la lutte actuelle et future contre le racisme. La délégation suisse a ainsi pu présenter son idée de créer un observatoire sur le racisme. Formée d'experts indépendants, cette institution serait chargée de contrôler l'application du plan d'action et de veiller à ce que ce texte ne reste pas lettre morte. La Suisse a également plaidé en faveur de la promotion d'institutions nationales pour lutter contre le racisme et pour la poursuite pénale d'actes et d'individus racistes. La délégation suisse tenait particulièrement à faire valoir les objectifs suivants: intensifier la lutte contre les sites Internet au contenu raciste, lutter contre la discrimination dans l'éducation et dans la formation, protéger les femmes contre une double discrimination, refuser d'assimiler le sionisme au racisme, assimiler l'antisémitisme au racisme, réserver l'emploi du terme «holocauste» uniquement pour l'extermination des Juifs sous le régime nazi, défendre les intérêts des Roms, des Yennish et des Sintis, exprimer des regrets au sujet de la traite des Noirs et de l'esclavage et, enfin, créer un forum pour débattre des différentes formes de réparations.

\section{$\square$ Influence de la conférence sur les affaires intérieures de la Suisse}

Les préparatifs pour la Conférence mondiale contre le racisme ont fourni l'occasion de relancer le débat sur l'antiracisme en Suisse. L'échéance a invité à dresser un bilan des sept ans de législation antidiscrimination, depuis l'introduction en 1994 de l'article 261 du Code pénal punissant l'incitation publique à la haine et à la discrimination raciales, qui constitue aujourd'hui encore le seul instrument de lutte antiraciste en Suisse. Le secrétaire de l'Association romande contre le racisme (ACOR) relève que cet instrument ne permet de combattre efficacement ni les manifestations de racisme au quotidien ni les formes de racisme institutionnel qui découlent de l'action de l'Etat. Et d'invoquer dès lors l'adoption d'une loi générale contre toute forme de discrimination.

De son côté, l'Association romande contre le racisme a adressé une pétition demandant aux Chambres fédérales de «refuser toute entrée en matière sur un projet de législation raciste et discriminatoire contraire à la Convention internationale de l'ONU sur l'élimination de la discrimination raciale». La pétition demande également au Conseil fédéral de retirer la réserve mise par la Suisse à l'application de la convention susmentionnée. Il s'agit de la critique majeure à l'encontre de la proposition de nouvelle loi sur les étrangers (voir début du sous-chapitre 5.4.2). Avec cette pétition, l'ACOR relance la proposition formulée par le Conseil de l'Europe lors de la Conférence européenne à Strasbourg en préparation à Durban, qui invite les Etats membres à abandonner toute réserve à l'égard de l'application de la convention onusienne.

C'est dans ce contexte que le Département fédéral de l'intérieur a créé un Secrétariat général chargé de la lutte contre le racisme et alloué un crédit de 15 millions de francs pour des projets en faveur des droits de l'homme et de la prévention contre le racisme, mettant ainsi en œuvre les mesures annoncées dans sa déclaration relative au rapport de la Commission indépendante d'experts SuisseSeconde Guerre mondiale ${ }^{1}$. 
Finalement, il convient de relever que la préparation de la Conférence de Durban a incité les Noirs habitant en Suisse à se regrouper en association, par delà les différentes appartenances nationales.

\section{$\square$ Déclaration finale et plan d'action}

A la dernière minute, au terme de vifs débats et après une prolongation de la conférence de toute une journée, les participants sont tout de même parvenus à adopter une déclaration finale commune et un plan d'action. Voici les principales conclusions et les appels de ces textes: l'esclavage et le trafic d'esclaves sont un crime contre l'humanité. Pour les participants à la conférence, ces deux méfaits constituent, avec le colonialisme, la principale source et la principale expression du racisme, de la discrimination raciale, de la xénophobie et des formes d'intolérance qui leur sont associées. Ce sont surtout les Noirs, les Asiatiques et les populations indigènes qui ont eu à les subir. La conférence regrette ces injustices infligées par le passé, dont les conséquences néfastes se font cependant sentir aujourd'hui encore. C'est pourquoi elle appelle les pays riches, l'ONU et les institutions financières internationales à trouver de nouveaux moyens pour lutter contre la pauvreté, contre le sous-développement et contre la marginalisation (p. ex. «Nouvelle Initiative africaine», cf. encadré à la fin de ce chapitre) et à appuyer ces efforts par des ressources financières accrues.

Les participants rejettent toute théorie raciste et condamnent les formes modernes de l'esclavage et du commerce d'êtres humains. Ils invitent instamment les Etats à prendre des mesures pour éradiquer ces pratiques, qui constituent des violations graves des droits de l'homme.

Les participants à la conférence ont fait part de leur grande préoccupation face à la situation au Proche-Orient et à la montée de l'antisémitisme, à l'islamophobie et à l'intolérance religieuse. Ils ont condamné les groupes racistes et violents qui s'en prennent aux communautés juives, arabes et musulmanes. La déclaration finale réaffirme le droit du peuple palestinien à l'autodétermination et à son propre Etat, de même que le droit à la sécurité de tous les Etats du ProcheOrient, Israël compris. Les participants ont par ailleurs rappelé que le souvenir de l'Holocauste doit rester vivant dans tous les esprits.

La conférence a invité les Etats à s'opposer à l'intolérance et à la discrimination religieuse. Elle a aussi appelé les pays impliqués dans le conflit au ProcheOrient à mettre fin à la violence et à reprendre les négociations de paix. Les participants ont réaffirmé que seuls des moyens pacifiques et le dialogue permettent de résoudre les conflits.

La conférence a reconnu les droits des populations indigènes ainsi que des minorités religieuses, ethniques ou culturelles, et a demandé l'abolition des discriminations institutionnelles et constitutionnelles dont ces groupes font l'objet. Elle a également demandé l'abolition des lois racistes qui peuvent faire partie de la politique migratoire et du droit pénal des Etats.

Enfin, la conférence a souligné que, malgré tout ce qui les sépare, chaque peuple et chaque individu appartiennent à une seule et même grande famille qui peuple la terre: l'humanité. 


\section{$\square$ Bilan de la conférence}

Sachant que la conférence a failli échouer, le compromis auquel elle est parvenue peut être considéré comme un succès, d'autant que les Etats africains et arabes, de même qu'Israël, se sont déclarés satisfaits du résultat final. Nombre de participants ont toutefois regretté que le problème du Proche-Orient ait occupé une telle place et qu'il ait relégué au second plan d'autres sujets non moins importants. La conférence ayant reconnu que le trafic d'esclaves constitue un crime contre l'humanité, elle permet de formuler des excuses officielles et d'envisager diverses formes de réparations. La prochaine fois que ce sujet sera soumis à la discussion internationale, il faudra cependant y inclure le commerce d'êtres humains pratiqué dans les Etats arabes et dans les pays africains. Il restera aussi à se mettre d'accord sur l'ampleur des conséquences néfastes de l'esclavage et du colonialisme, car on ne peut pas tenir le colonialisme pour seul responsable de tous les problèmes sociaux et économiques actuels.

Le plan d'action adopté au terme de la conférence formule une série d'incitations et de recommandations fort utiles. Si les divers Etats en tiennent compte pour les introduire dans leur législation nationale, alors la conférence n'aura pas été réunie en vain.

\section{SOURCES}

ACOR, <www.AcorSosRacisme.org>.

Conférence mondiale contre le racisme, la discrimination raciale, la xénophobie et l'intolérance qui y est associée, <www.un.org/french/WCAR/> et <www.unhcr.ch/html/racism>.

L'AGEFI, éditions du 29.9.01 au 10.9.01.

Le Courrier, éditions du 24.8.01 au 10.9.01.

Le Temps, «Les Noirs de Suisse s'associent pour mieux se défendre», 13.8.01; éditions du 25.8 .01 au 10.9.01.

Neue Zürcher Zeitung, éditions du 24.8.01 au 10.9.01.

\subsection{SESSION EXTRAORDINAIRE DE L'ASSEMBLÉE GÉNÉRALE DE L'ONU SUR LE VIH/SIDA}

Du 25 au 27 juin 2001, la communauté internationale s'est réunie à New York pour tenir une session extraordinaire sur le VIH/sida. Jamais dans leur histoire les Nations Unies n'avaient jusqu'alors consacré une session extraordinaire à une maladie. Le simple fait de tenir cette conférence a donc souligné l'acuité de ce problème mondial. La session avait pour objectif de renforcer la lutte internationale contre l'épidémie et de mobiliser les ressources nécessaires à cette lutte.

\section{$\square$ L'ampleur de l'épidémie}

Depuis que le VIH/sida a été identifié, en 1981, le syndrome d'immunodéficience acquise a tué 25 millions de personnes. De plus, sur les plus de 40 millions de personnes porteuses du virus à la fin de 2001, 5 millions ont été infectées au cours de cette année-là seulement. La région la plus touchée par la maladie est l'Afrique subsaharienne, où le nombre des personnes infectées était évalué à plus de 28 millions à la fin de 2001. Mais l'épidémie se répand rapidement aussi en Asie, en Europe de l'Est et dans les Caraïbes. Si certains pays ont réussi à freiner la propagation de la maladie, seul l'Ouganda est parvenu à réduire la proportion des personnes infectées par rapport à la population totale. 
L'augmentation des nouvelles infections enregistrée en Suisse en 2001 montre que la maladie est loin d'être vaincue, même dans les pays riches.

\section{$\square$ Les effets du VIH/sida sur le développement}

L'épidémie du sida compromet gravement le développement. En effet, la propagation rapide de la maladie constituera un sérieux obstacle à la réalisation de l'objectif arrêté à l'occasion du Sommet du millénaire, à savoir réduire de moitié le nombre des pauvres d'ici à 2015. Selon les estimations, la maladie peut aller jusqu'à ralentir de $1,2 \%$ la croissance annuelle des pays les plus touchés. L'épidémie accroît ainsi le nombre des pauvres et réduit l'espérance de vie (de vingt ans dans certains pays). Les gouvernements et les entreprises perdent des employés, mais aussi les compétences que ceux-ci avaient acquises. Les coûts - dans les domaines social, de la sécurité sociale et de la formation engendrés par l'épidémie dépassent largement les moyens dont disposent les pays les plus atteints et réduisent à néant des progrès chèrement acquis. L'agriculture étant également privée de ressources et de main-d'œuvre, la production vivrière tend à diminuer dans certaines régions.

Ce sont surtout les femmes et les jeunes filles qui font les frais de cette situation. En effet, alors que les femmes se chargent de soigner les proches tombés malades, les jeunes filles doivent assumer de plus grandes responsabilités au sein de la famille et n'achèvent pas leur scolarité. Le sida affaiblit toute la structure de la société et représente une menace pour la stabilité politique et sociale.

L'expérience des pays où la lutte contre la maladie s'avère relativement efficace montre que le seul moyen de préserver les acquis du développement consiste à intégrer la lutte contre le sida dans les stratégies et dans les programmes de développement. Pour endiguer efficacement la maladie, il importe d'intégrer toutes les institutions de l'administration gouvernementale et de la société civile dans un programme national unique et de fournir une formation adéquate aux personnes qui œuvrent dans les domaines concernés.

\section{$\square$ Déclaration finale}

Au terme de vifs débats, l'Assemblée générale a trouvé un consensus et adopté une déclaration d'engagement intitulée $A$ crise mondiale, action mondiale. Elle y constate que la pauvreté et l'«ignorance» du risque sont les principaux facteurs qui contribuent à la propagation du sida; elle souligne aussi que nier ou minimiser le problème, voire marginaliser et discriminer les malades, c'est aller à l'encontre des efforts de prévention et aggraver les effets de l'épidémie.

Le premier plan mondial de lutte contre le VIH/sida se donne les priorités suivantes: développer la prévention, améliorer l'accès aux soins et au traitement, prendre en charge les orphelins du sida, élargir le partenariat entre les secteurs public et privé, promouvoir une action multisectorielle plus rapide face à l'épidémie et mobiliser des ressources correspondant à la gravité de la crise.

Pour ce qui est des ressources, le plan demande à la communauté internationale d'investir pendant dix ans au minimum 7 à 10 milliards de dollars par an dans les pays à revenu faible ou moyen (1,5 à 2 milliards de dollars y ont été dépensés pour la lutte contre le sida jusqu'ici). Une partie de l'argent devrait provenir des pays touchés eux-mêmes, tandis que le reste devrait être fourni par d'autres 
sources (pays riches, secteur privé, organisations internationales, ONG). Si les moyens mis à disposition n'atteignent pas le niveau souhaité, le traitement et la prise en charge des malades ne pourront pas être assurés. Or les experts jugent indispensable d'appliquer une double stratégie incluant aussi bien la prévention que le traitement.

La mention de groupes à risques (les prostituées et leurs clients, les toxicomanes, les homosexuels) aurait pu servir de base à l'élaboration de programmes de prévention spécifiques. Divers Etats religieux et des pays fortement influencés par des groupements religieux conservateurs ont toutefois exercé de fortes pressions pour que ces groupes ne figurent pas expressément dans la déclaration. Celle-ci souligne néanmoins que le respect des droits de l'homme, l'égalité entre hommes et femmes et l'arrêt de la violence à l'égard des femmes constituent une base solide pour prévenir efficacement le sida.

La délégation suisse à cette session extraordinaire était emmenée par la conseillère fédérale Ruth Dreifuss. Celle-ci a appelé la communauté internationale à accroître les moyens destinés à lutter contre le virus de l'immunodéficience humaine. Pour souligner son propos, elle a annoncé qu'entre 2000 et 2004 la Suisse allait quadrupler, c'est-à-dire faire passer à 8 millions de francs, la contribution qu'elle verse à ONUSIDA et aux autres institutions internationales qui luttent contre le sida. La conseillère fédérale a par ailleurs plaidé en faveur d'une nette baisse des prix des médicaments contre le sida.

\section{$\square$ Fonds mondial de lutte contre le sida, la tuberculose et le paludisme ${ }^{2}$}

Ce fonds, qui devra disposer de plusieurs milliards de dollars, servira à financer la lutte contre le sida, la tuberculose et le paludisme. Dans un discours à l'occasion de l'assemblée de l'Organisation mondiale de la santé, Kofi Annan a souligné que les conséquences dévastatrices de ces maladies comptent parmi les principaux obstacles au développement.

Le fonds, qui devra être établi à la fin de 2001, sera dirigé par un organe indépendant; il ne doit ni remplacer les mécanismes de financement qui alimentent les programmes contre le sida, la malaria et la tuberculose, ni supplanter les efforts et les investissements des pays en développement. Le fonds sera donc constitué par des moyens nouveaux et complémentaires. Tous les Etats, les entreprises et les particuliers ont été invités à participer son financement. Les promesses de financement réunies jusqu'à la fin de 2001 totalisaient près de 1,5 milliard de dollars. Une vaste campagne de collecte de fonds sera organisée en 2002.

\section{$\square$ L'accès aux médicaments en question}

Bien que la politique des prix ne soit que l'un des nombreux aspects de la lutte contre le sida, le prix des médicaments est souvent au centre des débats. Ceux-ci mettent particulièrement notre pays en cause puisque deux des plus grandes multinationales pharmaceutiques ont leur siège en Suisse.

Dans les pays industrialisés, le traitement médicamenteux d'une personne infectée par le virus atteint, à lui seul, près de 12’000 francs suisses par an. Le

2. Selon les dernières informations, le nom officiel du fonds est le suivant: «Global Fund to Fight Aids, Tuberculosis and Malaria » (GF-ATM). 
traitement des 40 millions de personnes séropositives de par le monde engloutirait donc des sommes astronomiques. Pour les pays pauvres, il est dès lors vital de pouvoir obtenir des médicaments à des prix nettement plus bas ${ }^{3}$. Le débat intervient à deux niveaux: d'une part, on souhaite que les multinationales pharmaceutiques accordent une baisse des prix; d'autre part, on envisage la possibilité de produire des génériques bon marché dans les pays du Sud (pour les exporter vers d'autres pays du Sud). Or ce débat s'inscrit dans une controverse plus large, celle autour de la protection des brevets, qui est régie par 1'Accord sur les ADPIC dans le cadre de l'OMC.

ASTM 2002, sous-chapitre 2.4.

De leur côté, les sociétés pharmaceutiques avancent toutes sortes d'arguments pour s'opposer aux solutions qui pourraient réduire leurs bénéfices. Elles prétendent par exemple qu'une utilisation inappropriée des médicaments peut amener le virus à développer des résistances ou soulignent le coût du développement de futurs médicaments: si la protection des brevets perd trop de sa substance, il n'y aurait plus aucun intérêt à investir dans la recherche et dans le développement de nouvelles préparations. Les entreprises pharmaceutiques sont néanmoins sous pression et certaines sont prêtes à accepter des compromis.

SOURCES

Fonds mondial de lutte contre le SIDA, la tuberculose et le paludisme, <www.unfoundation.org/ campaigns/aids $>$ (en anglais).

NGLS Roundup, $\mathrm{n}^{\circ} 76$, août 2001.

Programme commun des Nations Unies sur le VIH/sIDA, <www.unaids.org>.

Session extraordinaire de l'Assemblée générale sur le VIH/sIDA, <www.un.org/french/ga/sida/ conference/>.

L'AGEFI, 28.6.01.

Le Courrier, 22.6.01.

Le Temps, 22.6.01.

\subsection{CALENDRIER DES CONFÉRENCES INTERNATIONALES}

\section{$\square 57^{e}$ Session de la Commission des droits de l'homme}

La $57^{\text {e }}$ Session de la Commission des droits de l'homme s'est tenue à Genève du 19 mars au 27 avril 2001. Dans la perspective de la Conférence mondiale contre le racisme, des débats sur la tolérance et sur le respect étaient à l'ordre du jour. Au premier jour de la session, le haut-commissaire aux droits de l'homme, Mary Robinson, a d'emblée annoncé qu'elle n'était pas disposée à assumer un deuxième mandat. Pour expliquer son retrait, elle a invoqué le manque de retenue des acteurs en présence et le manque de ressources mises à la disposition du haut-commissariat. En effet, moins de $2 \%$ du budget de l'ONU sont consacrés à la promotion des droits de l'homme.

La commission a examiné la situation des droits de l'homme dans 22 pays et régions, se penchant en priorité sur les Etats où des conflits armés mettaient ces droits à rude épreuve.

3. Même si les prix étaient fixés au plus bas (si le traitement d'une personne coûtait par exemple 1 franc suisse par jour), le risque subsisterait que la lutte contre le sida soit menée au détriment d'autres tâches qui incombent à la santé publique. 
L'opposition entre les pays industrialisés et les pays en développement est apparue au grand jour lors de l'adoption de diverses résolutions spécifiques: tandis que les Etats occidentaux voudraient avant tout renforcer les droits politiques fondamentaux, les pays du tiers-monde souhaitent renforcer le droit au développement. Les résolutions adoptées concernent notamment les droits de l'homme et la mondialisation; le droit au développement, au logement, aux médicaments contre le sida et à l'éducation; les droits des femmes et des enfants; ainsi que le droit à l'alimentation.

Le mandat du Suisse Jean Ziegler, rapporteur spécial sur le droit à l'alimentation, a été étendu pour englober également le droit à l'eau.

Il convient aussi de relever une résolution qui prévoit de nommer un rapporteur spécial sur les peuples indigènes et une résolution sur l'abolition de la peine de mort.

Lors de l'élection des nouveaux membres de la commission, les Etats-Unis n'ont pas été réélus. C'est la première fois depuis la création de la commission, en 1947, que ce pays n'en sera pas membre.

SOURCES

Haut-Commissariat aux droits de l'homme, <www.unhchr.ch>.

$57^{\mathrm{e}}$ Session de la Commission des droits de l'homme, <www.unhchr.ch/french/html/menu2/2/57chr/ 57main_fr.htm>.

Neue Zürcher Zeitung, 20.3.01, 20.4.01, 28.4.01.

Le Temps, 19.4.01, 28.4.01.

\section{$\square$ Assemblée mondiale de la santé (OMS)}

La $54^{\text {e }}$ Assemblée mondiale de la santé s'est tenue à Genève du 14 au 22 mai 2001. Les principaux sujets à l'ordre du jour comprenaient la lutte contre le sida, l'alimentation des nourrissons, les moyens d'endiguer la consommation de tabac ainsi que la mobilisation des Etats en faveur d'une promotion active de la santé. La directrice générale de l'Organisation mondiale de la santé, Gro Harlem Brundtland, a lancé un vibrant appel pour que le monde surmonte les maladies provoquées et entretenues par la pauvreté.

L'assemblée a adopté une résolution sur l'accès aux médicaments, dans laquelle elle a souligné qu'un tiers de la population mondiale n'a pas accès aux médicaments de base indispensables. Elle préconise d'instaurer un contrôle des prix pour combler cette grave lacune et souhaite que l'on encourage les scientifiques à mettre au point des médicaments plus efficaces contre les maladies qui touchent avant tout les populations pauvres.

Une longue résolution consacrée à l'alimentation des nourrissons et des jeunes enfants invite par ailleurs les 191 Etats membres à promouvoir l'allaitement exclusif du nourrisson au cours des six premiers mois.

Les pays membres ont aussi lancé un appel à plus de vigilance à l'égard de l'industrie du tabac, qui a sapé pendant des années les efforts des gouvernements et de l'OMS pour lutter contre le tabagisme. Il s'agit aussi d'améliorer la réglementation - encore lacunaire - qui régit les produits à base de tabac et qui a pour objectif de protéger la santé publique.

Représentée au sein du Conseil exécutif jusqu'en 2002, la Suisse a pu contribuer activement à la préparation des sujets à l'ordre du jour dans le cadre de la Conférence préparatoire. 
La délégation suisse, emmenée par Ruth Dreifuss, s'est notamment engagée dans la lutte contre le tabagisme et pour la réforme du Conseil exécutif. Sa proposition visant à augmenter la transparence des contrôles sur le tabac ayant soulevé de vives oppositions de la part d'autres délégations, la Suisse a renoncé à en faire officiellement état. Sur l'initiative de la Suisse, un paragraphe a par ailleurs été biffé du projet de résolution sur l'accès aux médicaments. Ce paragraphe - qui aurait chargé l'OMS d'encourager la production locale de médicaments - aurait contrevenu à l'Accord sur les ADPIC de l'OMC.

SITE INTERNET

Organisation mondiale de la santé: <www.who.int>.

\section{$\square$ Conférence internationale du travail}

La $89^{\text {e }}$ Session de la Conférence internationale du travail s'est tenue à Genève du 5 au 21 juin 2001. Cette conférence annuelle de l'Organisation internationale du travail (OIT) est l'occasion de débattre de problèmes sociaux et professionnels à l'échelle mondiale. La conférence est également compétente pour adopter les normes internationales sur le travail, l'orientation politique et le budget de l'OIT. Selon J.-J. Elmiger, ambassadeur suisse, l'Organisation internationale du travail joue le rôle de «protectrice de la dimension sociale de la mondialisation ».

Chaque Etat membre peut envoyer quatre délégués à la conférence (deux délégués du gouvernement, un délégué représentant les employeurs et un délégué représentant les travailleurs). En 2001, la conférence a notamment abordé les points suivants: élimination de toute forme de travail forcé ou obligatoire, sécurité et santé dans l'agriculture, promotion des coopératives et avenir de la sécurité sociale.

Dans son rapport à la conférence, le directeur général de l'OIT, Juan Somavia, a souligné combien il était important de développer les activités en faveur d'un «travail décent». A son avis, il s'agit désormais de quitter le domaine des aspirations et de la théorie pour passer aux actes et mettre en œuvre les mesures prévues. La mise en place de conditions de travail plus humaines permettrait en effet de lutter contre la pauvreté et fournirait une plus grande légitimité à l'économie mondiale. La Suisse plaide également en faveur d'un tel programme et pour la mise à disposition des moyens nécessaires à sa réalisation. Pour les années 2002 et 2003, elle va donc augmenter de 5\% sa contribution à l'OIT. Cette contribution se montera ainsi à près de 4 millions de francs, ce qui équivaut à $1,3 \%$ du budget de l'OIT.

La séance spéciale consacrée à l'application des conventions a examiné le cas de 24 pays. Outre des progrès réalisés au Portugal et en Turquie, on a aussi relevé d'importants manquements: le Bélarus, la Colombie, le Venezuela et l'Ethiopie violent la Convention $n^{\circ} 87$ sur la liberté syndicale et sur la protection du droit syndical. De plus, le travail forcé étant largement répandu au Myanmar et au Soudan, ils ne respectent pas la convention $n^{\circ} 29$, qui interdit toute forme de travail forcé. Accroître la pression sur le Myanmar devrait inciter ce pays à se montrer plus respectueux.

La conférence a pour la première fois adopté des normes internationales sur la sécurité et sur la santé dans l'agriculture. Il faut savoir que l'agriculture compte, avec l'exploitation minière et la construction, parmi les secteurs d'activité les 
plus dangereux. A tel point que la moitié des 1,2 million de décès annuels dus à des accidents du travail sont enregistrés dans l'agriculture. La plupart des accidents surviennent lors de la manutention de produits chimiques toxiques ou lors du maniement de machines.

Les principaux éléments de la nouvelle convention comprennent la définition de moyens appropriés pour évaluer les risques; des mesures de prévention et de protection pour garantir la sécurité lors du travail avec des machines, avec des produits chimiques et avec des animaux, pour garantir la sécurité de l'entreposage et du transport, et pour garantir la sécurité dans la construction et dans l'entretien d'installations agricoles; et une assurance pour les travailleurs agricoles en cas d'accident, de maladie ou de décès. Cette convention ne s'applique toutefois qu'aux employés agricoles et non aux agriculteurs indépendants. Les délégués suisses ont approuvé cette convention adoptée à la grande majorité des participants.

Dans le cadre du débat sur une convention internationale pour la promotion de coopératives autonomes, on a souligné que de telles coopératives jouent un rôle important dans la création d'emplois. Ces coopératives peuvent aussi faciliter grandement la recherche de capitaux, tout en favorisant l'entraide et la création de réseaux sociaux.

Un système national de sécurité sociale et d'assurance maladie est au centre des préoccupations de nombreux pays membres. La plupart d'entre eux sont unanimes pour déclarer que la politique sociale doit avoir pour objectif d'étendre cette protection à l'ensemble de la population. En effet, une assurance maladie et une sécurité sociale nationales contribuent à augmenter la productivité et se révèlent dès lors utiles pour le développement économique.

Dans ce cadre, les pays industrialisés se préoccupent avant tout du financement du système des retraites alors que leurs populations ne cessent de vieillir, tandis que nombre de pays en développement doivent surtout faire face aux problèmes liés à l'épidémie du sida.

Le Programme international pour l'abolition du travail des enfants (IPEC) - que la Suisse finance depuis 1997 avec 24 autres Etats donateurs - a lancé une nouvelle initiative dans le but d'éliminer en l'espace de dix ans les pires formes du travail des enfants (tri des déchets, transport de charges, travail dans les mines, travail dans la pêche et dans l'agriculture commerciales, prostitution et servitude pour dettes) dans trois pays: Tanzanie, Salvador et Népal. D'autres pays devraient également se joindre à cette initiative.

Le Conseil fédéral a fait savoir que la Suisse n'était pas en mesure de ratifier la Convention $n^{\circ} 183$ sur la protection de la maternité, adoptée à l'occasion de la dernière session de la conférence, car la législation suisse ne garantit pas le minimum de protection prévu dans cette convention.

凹 ASTM 2000, pp. 204-205.

\section{SOURCES}

Conférence internationale du travail $-89^{\mathrm{e}}$ session, <www.ilo.org/public/french/standards/relm/ilc/ilc89/ index.htm>.

NGLS Go Between, «89 $9^{\text {th }}$ International Labour Conference», $\mathrm{n}^{\circ}$ 87, Genève, juillet-août 2001.

Organisation internationale du travail, <www.ilo.org $>$. 
Suivi de la Conférence des Nations Unies sur les établissements humains (Istanbul +5)

Du 6 au 8 juin 2001, cinq ans après la Conférence sur les établissements humains (Habitat II), l'Assemblée générale des Nations Unies s'est réunie à New York en une session extraordinaire, intitulée «Istanbul +5 ».

A son issue, Habitat II avait marqué un changement fondamental en matière d'urbanisation: en effet, au lieu de chercher à ralentir l'urbanisation - objectif utopique en soi - on avait décidé de viser à mieux la planifier.

ASTM 1997, pp. 66-72.

Cinq ans après l'adoption du plan d'action sur l'habitat «Un logement convenable pour tous», il s'agissait de faire le bilan des activités menées pendant ce laps de temps: comment a-t-on mis en œuvre les préoccupations du plan d'action? Quels obstacles a-t-on rencontrés lors de sa réalisation? Quelles nouvelles actions fallait-il prévoir? L'évaluation des progrès accomplis s'est fondée sur des rapports nationaux, dans lesquels les gouvernements ont consigné ce qui a été entrepris dans leur pays.

Istanbul +5 n'a pas permis de faire des progrès spectaculaires. Voici en effet les principaux résultats de la conférence: confirmation des engagements d'Habitat II, débats sur les principaux problèmes de l'urbanisation et adoption de la «Déclaration sur les villes et d'autres établissements humains en ce nouveau millénaire».

Depuis 1996, l'urbanisation s'est encore accélérée; les inégalités entre les villes et à l'intérieur même des villes se sont accentuées et la qualité de vie en ville tend à se détériorer. La déclaration finale traite surtout de la pauvreté urbaine qui ne cesse de s'accentuer et constate que de nouveaux moyens et de nouvelles actions sont nécessaires, surtout dans les pays du tiers-monde, pour faire face à ce problème.

L'autonomie des communes a été l'un des principaux sujets de débat et la conférence a reconnu que des structures décentralisées favorisent le développement. Un projet visant à faire adopter une charte internationale de l'autonomie locale a toutefois été rejeté, car la majorité des participants ont jugé qu'une telle charte s'immiscerait par trop dans la politique intérieure des Etats.

La délégation suisse a exprimé ses regrets de voir que les Nations Unies n'acceptent que très mal la notion de partenariat (intégration d'intervenants privés et de la société civile dans les processus de prise de décision au niveau politique), pourtant qualifiée de novatrice à Istanbul. Elle a souligné qu'un tel partenariat intervient souvent au niveau local, mais que les propositions et les décisions à un niveau supérieur proviennent principalement du «Club des nations» et excluent largement la société civile. Pour la Suisse, les oppositions formulées au cours de cette conférence à l'implication de la société civile sont donc en contradiction avec les décisions prises à Istanbul et constituent un retour en arrière. Selon la délégation suisse, le principal défi qu'il s'agira de relever ces prochaines années consistera à réduire le fossé entre les décisions internationales et les actions concrètes sur le plan local, pour parvenir à une plus grande cohérence et à une plus grande efficacité du développement urbain.

Les organisations non gouvernementales ont été déçues de constater que la déclaration finale n'évoque pas du tout le droit à «un logement convenable pour 
tous ». Pour elles, cette omission équivaut à un net recul par rapport aux résultats d'Habitat II.

SOURCES

Centre des Nations Unies pour les établissements humains (CNUEH), <www.unchs.org >.

NGLS Roundup, «Istanbul+5: UN General Assembly Review of the Habitat Agenda», n 79, Genève, août 2001.

Session extraordinaire de l'Assemblée générale des Nations Unies Istanbul+5, <www.un.org/french/ga/ istanbul5/ga/habitat/>.

\section{$\square$ Session annuelle du Conseil économique et social des Nations Unies}

La Session annuelle du Conseil économique et social des Nations Unies s'est tenue à Genève du 2 au 27 juillet 2001. Chargé de coordonner les activités économiques et sociales de l'ONU, le conseil est l'un des principaux organes de l'organisation. Il se compose de 54 membres élus pour trois ans.

Lors de cette session, le conseil s'est surtout penché sur l'aide internationale à l'Afrique. Les Nations Unies ont en effet constaté qu'au cours des années 1990 les guerres, l'épidémie du sida et le poids de la dette ont anéanti les progrès réalisés en Afrique au cours des années 1980. Qui plus est, même l'aide internationale a connu un net recul dans les années 1990.

Pour réaliser l'objectif fixé l'année précédente à l'occasion du Sommet du millénaire - à savoir réduire de moitié le nombre des êtres humains vivant dans la pauvreté d'ici à 2015 - l'Afrique aurait besoin de suivre une croissance annuelle de l'ordre de 7 à $8 \%$. Or il apparaît impossible d'atteindre cet objectif sans améliorer de manière spectaculaire le contexte global. Le secrétaire général des Nations Unies, Kofi Annan, a donc demandé la tenue d'une nouvelle ronde de négociations sur le commerce mondial, qui serait consacrée aux besoins des pays les plus pauvres, et il a souligné à quel point il est important que les marchés du Nord s'ouvrent aux produits africains. La déclaration adoptée au terme de la session appelle les Nations Unies et la communauté internationale à soutenir la « Nouvelle Initiative africaine».

\section{Nouvelle Initiative africaine}

Réunis à Abuja, la capitale du Nigeria, en octobre 2001, plusieurs chefs d'Etat et de gouvernement africains ont lancé le "Nouveau Partenariat pour le développement de l'Afrique» (New Partnership for African Development, NEPAD). Désormais plus connu sous le nom de "Nouvelle Initiative africaine», ce plan ambitieux a été élaboré par les présidents du Nigeria, de l'Afrique du Sud, du Sénégal et de I'Algérie, avant d'être présenté au sommet de l'Organisation de l'unité africaine à Lusaka et au sommet du G8 à Gênes. Ce plan vise à vaincre la pauvreté en mettant l'Afrique sur la voie d'une croissance rapide et d'un développement durable. A cet effet, le plan prévoit d'importants investissements dans les domaines de l'éducation, de l'infrastructure et de l'informatique, ainsi que des efforts communs pour faire régner la paix et pour instaurer la démocratie. Le plan innove notamment parce qu'il a été lancé par des dirigeants africains désireux de prendre eux-mêmes l'initiative et parce qu'il met l'accent sur le partenariat et non pas sur l'aide. La prochaine étape concrète du plan comprend la création d'un secrétariat du NEPAD, à Pretoria, et la mise sur pied d'un comité exécutif.

Sources: Neue Zürcher Zeitung, 25.10.01; "New Partnership for African Development (NEPAD) Incorporating the OMEGA Plan n, <www.mapstrategy.com>. 
SOURCES

Conseil économique et social, $<w w w . u n . o r g / f r e n c h / d o c u m e n t s /$ ecosoc/.htm $>$ et $<w w w . u n . o r g /$ esa/coordination/ecosoc>.

Le Courrier, 3.7.01.

NGLS Roundup, $\mathrm{n}^{\circ} 77$, août 2001.

\section{$\square$ Conférence internationale de l'éducation}

La Conférence internationale de l'éducation, organisée par le Bureau international de l'éducation (IBE) de l'UNESCO, s'est tenue à Genève du 5 au 8 septembre 2001. La conférence avait été placée sous le slogan «Apprendre à vivre ensemble» et s'est principalement penchée sur les contenus et sur les stratégies de l'enseignement, sur les problèmes qu'ils suscitent et sur les solutions à leur apporter. En bref, puisque des progrès ont été obtenus sur le plan quantitatif (scolarisation du plus grand nombre d'enfants), il s'agit à présent d'accroître la qualité de l'enseignement.

Pour apprendre à vivre ensemble, il importe de faire régner un climat de tolérance et de respect réciproque dans les écoles. Après de vives discussions dans le cadre d'ateliers à thème et en session plénière, le rapport qui a finalement pu être adopté souligne que les progrès scientifiques et techniques, la coexistence des cultures et la mondialisation placent les acteurs du secteur de l'éducation et de la formation face à d'énormes défis. De plus, les réformes à mener doivent être perçues comme des processus et non pas comme des résultats atteints une fois pour toutes.

Conscients du fait que les problèmes sociaux ne sont pas du seul ressort de l'école, les délégués ont invité tous les acteurs de la société à dialoguer et à collaborer pour résoudre ces problèmes.

\section{SOURCES}

Bureau international d'éducation, <www.ibe.unesco.org $>$.

$46^{\mathrm{e}}$ session de la Conférence internationale de l'éducation, <www.ibe.unesco.org/International/ICE/ 46francais/46menuf.htm>. 